\title{
Pulsations of Luminous Blue Variables
}

\author{
E. A. Dorfi ${ }^{1}$, A. Gautschy ${ }^{2}$ \\ ${ }^{1}$ Institut für Astronomie, Türkenschanzstr. 17, A-1180 Wien, Austria, \\ dorfi@astro.univie.ac.at \\ ${ }^{2}$ ETH-Bibliothek, Rämistr. 101, Ch-8092 Zürich, gautschy@library.ethz.ch
}

\begin{abstract}
From simulations of massive stars we find regular low-amplitude radial pulsations can be excited. Such pulsations were encountered during core hydrogen burning as well as during the early core helium burning stage of evolution. For four selected models we present light curves in the V-band. The results are discussed with the aim to guide observations to identify and monitor such regularly pulsating variable massive stars in nature.
\end{abstract}

\section{Introduction}

The COROT mission offers the possibility for high-precision measurements of photometric variations of stars. With increasing accuracy more and more domains with light variability are being identified on the Hertzsprung-Russell (HR) diagram. This applies in particular to the high-luminosity regime where the luminous blue variables (LBVs) are located. The results from the HIPPARCOS mission (Eyer \& Grenon 1997) demonstrated that almost all stars with luminosity class lower than III show noticeable light variability (above some $10 \mathrm{mmag}$ ) of some as yet unknown origin.

From the observational point of view the nature of LBV variability is still not well understood. On timescales of years and months, eruption-like outbursts have been reported but small amplitude, quasi-regular photometric variations have also been detected on timescales of several days (e.g. Nota \& Lamers (1997) for a summary on LBV properties). Due to the small number of known LBVs (see Table 1) and the insufficient observational coverage, the physical nature of either the micro-variability or the outbursts is not clear up to date. Based on a search through the literature the latest discovery of a new LBV 
Table 1: Known luminous blue variables (after Bohannan 1997 and Shemmer et al. 2000)

\begin{tabular}{lll}
\hline Galaxy & $\#$ & LBVs \\
\hline Milky Way & 5 & $\eta$ Car, P Cyg, AG Car, HR Car, HD160529 \\
LMC & 6 & S Dor, R71, R127, HD269582, R110, R143 \\
SMC & 1 & R40 \\
M31 & 4 & AE And, AF And, Var A-1, Var 15 \\
M33 & 4 & Var B, Var C, Var 2, Var 38, B416 \\
NGC2403 & 5 & V12, V22, V35, V37, V38 \\
M81 & 3 & I1, I2, I3 \\
M101 & 3 & V1, V2, V10 \\
NGC1058 & 1 & SN1961V \\
\hline
\end{tabular}

has been reported by Shemmer et al. (2000) for the star B416 located in M33. Hence, photometric satellite missions like COROT could offer a unique opportunity to gain new insights into the physical mechanism operating in the most luminous stars.

The theoretical results of the LBV-phase have been strongly debated and the suggested models range from strange mode instabilities (Kiriakidis et al. 1993) to an oscillatory dynamically unstable pulsation for stars near to the Eddington limit (Stothers \& Chin 1993). However, none of the models presented are able to explain the micro-variability, the eruptive outbursts or the stellar winds also observed during the LBV phase. According to Leitherer (1997) the observationally deduced mass loss rates for LBVs not undergoing a major outburst range from $10^{-4} \mathrm{M}_{\odot} \mathrm{yr}^{-1}$ for $\eta$ Car to $6 \cdot 10^{-7} \mathrm{M}_{\odot} \mathrm{yr}^{-1}$ for $\mathrm{R} 71$ (see also Table 1 ). The final outflow velocities are typically around $200 \mathrm{~km} / \mathrm{s}$.

In this short contribution we want to focus on the most luminous stars, the so-called LBVs (luminous blue variables) or S Dor variables. Since the observed number of such variables is rather small, theoretical predictions of the variability become more important.

We have performed radial non-linear pulsation computations for these stars by applying the full set of the radiation hydrodynamics equations (RHD, e.g. Mihalas \& Mihalas 1984). The initial models are computed from usual stellar evolution calculations where the corresponding evolutionary paths are plotted in Fig. 1. The pulsational models investigated belong to massive stars at the stage of either core hydrogen buring or early core helium burning. The numerical method used for the non-linear pulsation simulations is described in detail in Dorfi (1998). In the dynamical computations the radiative transfer is performed by using the Rosseland-mean OPAL92-opacity for the radiative flux and 
Table 2: Table of LBV model parameters and pulsation properties

\begin{tabular}{|c|c|c|c|c|c|c|c|c|}
\hline Name & $\begin{array}{c}M \\
{\left[\mathrm{M}_{\odot}\right]}\end{array}$ & $\begin{array}{c}L \\
{\left[10^{3} \mathrm{~L}_{\odot}\right]}\end{array}$ & $\begin{array}{l}T_{\text {eff }} \\
{[\mathrm{K}]}\end{array}$ & $\begin{array}{c}P \\
\text { [days] }\end{array}$ & $\Delta R / R$ & $\begin{array}{c}\Delta u \\
{[\mathrm{~km} / \mathrm{s}]}\end{array}$ & $\begin{array}{c}u_{\mathrm{s}} \\
{[\mathrm{km} / \mathrm{s}]}\end{array}$ & $\begin{array}{l}\Delta m_{\mathrm{V}} \\
{[\mathrm{mag}]}\end{array}$ \\
\hline M20C & 20 & 66 & 27100 & 0.290 & 0.033 & 63 & & 0.11 \\
\hline M45 & 45 & 537 & 33890 & 0.575 & 0.080 & 248 & 48 & 0.11 \\
\hline $\mathrm{M} 60 \mathrm{H}$ & 60 & 933 & 34680 & 1.611 & 0.368 & 598 & 211 & 0.27 \\
\hline M60C & 60 & 900 & 18000 & 4.086 & 0.281 & 356 & 114 & 0.26 \\
\hline
\end{tabular}

the Planck-mean for the corresponding radiation energy equation. The calculated profiles (density, temperature, etc.) are taken as input for a frequencydependent radiative transfer. After folding the obtained spectra with the filter passbands we get e.g. the V-light curves presented in Fig. 1. To enhance the structural differences the areas below the observable light curves have been filled with grey colour.

\section{Theoretical light curves of LBVs}

The properties of the LBV models discussed in this paper are summarized in Table 2. The models exhibit rather regular pulsations (cf. Fig. 1) with periods $P$ shown in column 5 . The relative changes of the photospheric radius $\Delta R / R$ are given in column 6 indicating that only the masses above $30 \mathrm{M}_{\odot}$ show pronounced radial oscillations with velocity variations $\Delta u$ (column 7 ). These motions go along with strong shock waves running through the atmosphere and the shock speed $u_{\mathrm{s}}$ is given in column 8 . Finally, the last column states the changes in the visual magnitude $\Delta m_{\mathrm{V}}$, which allows a direct comparison with observed LBVs. According to the results we emphasize the non-adiabatic nature of the pulsations, where large velocity amplitudes are accompanied by small luminosity variations of the order of about $0.1 \mathrm{mag}$. For light curves in different passbands we refer to Dorfi \& Gautschy (2000). Due to the dominance of the radiation pressure these oscillations are mainly confined to the outer layers. From a comparison with linear stability analysis they seem to be rather regular radial modes (for a review e.g. Gautschy \& Saio 1995).

The temporal evolution of a pulsating star (model: $\mathrm{M} 60 \mathrm{H})$ is plotted in Fig. 2 through the motion of different mass shells over two pulsational cycles. The period of the outer (observable) shell is $P=1.611$ days. In contrast to this periodicity the inner shells are oscillating with half of that period, namely $P=0.805$ days. This short period corresponds exactly to the pulsation period obtained from a linear stability analysis which causes also the most prominent 

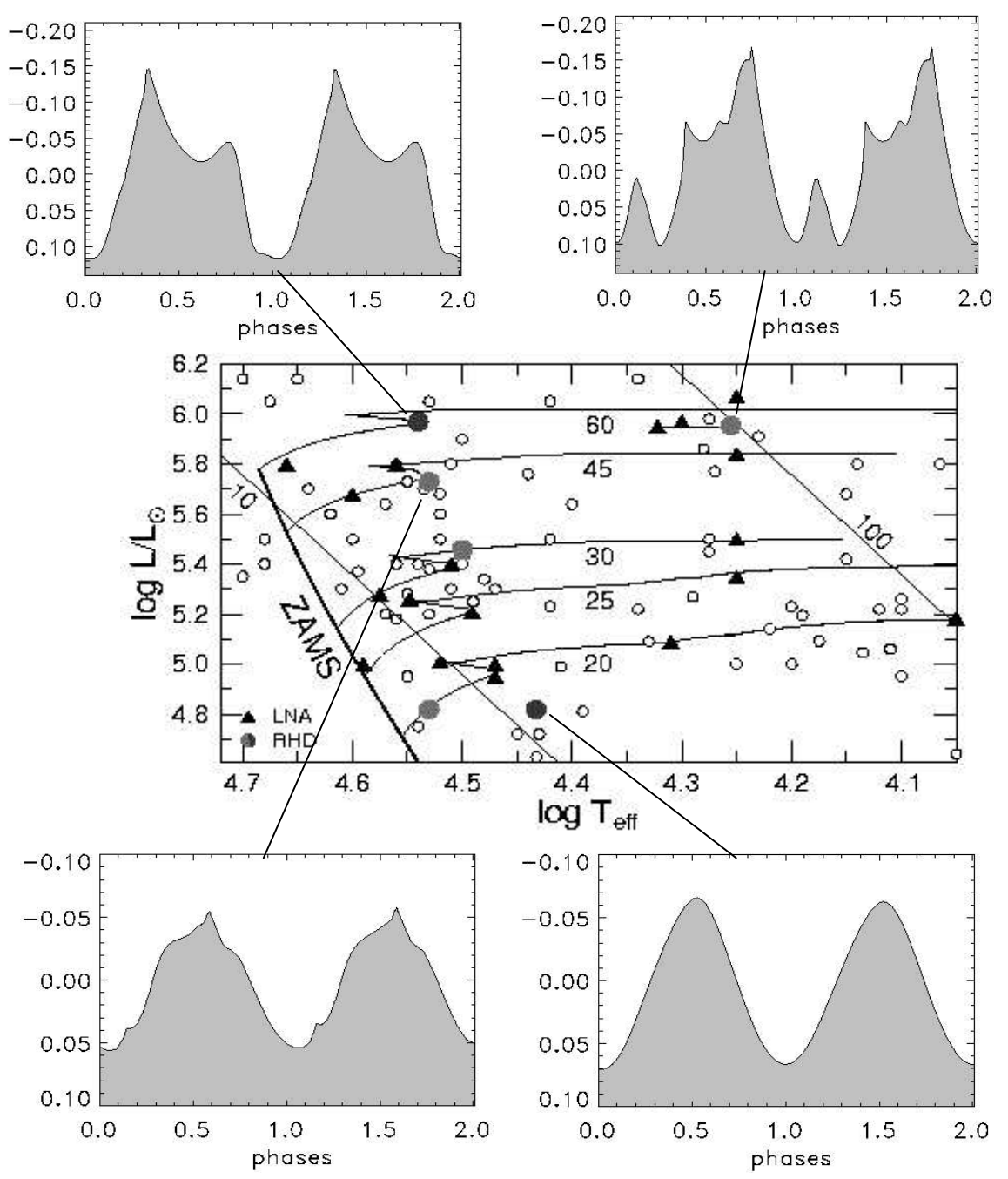
peak in a power spectrum of the inner mass shells (Dorfi \& Gautschy 2000). However, the non-linear reactions of the outermost layers induce a longer periodicity visible for an external observer because the motions of the upper atmosphere are dominated by two strong shock waves seen at phases 0.35 and 0.7 . These outward running waves travel at speeds of $u_{\mathrm{s}}=136 \mathrm{~km} / \mathrm{s}$ and $u_{\mathrm{s}}=211 \mathrm{~km} / \mathrm{s}$. The Mach numbers of these shocks vary between 5 and 8 . After the acceleration event the mass shells are moving basically along ballistic trajectories characterized by the dynamical time scale of the stellar envelope. Clearly, such shock compressions heat the thermal gas and the corresponding features are imprinted on the light curves. Due to the low optical depth in the outer layers no phase shift of the features is introduced in the light curves if observed in different passbands.

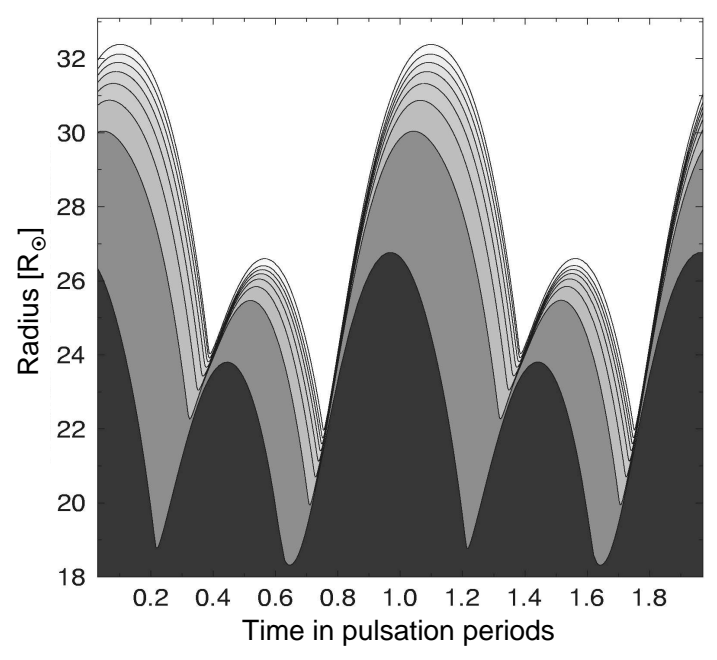

Figure 2: The paths of different mass shells as a function of the pulsation period show the ballistic behavior of the outermost atmosphere $\left(M=60 \mathrm{M}_{\odot}, L=933000 \mathrm{~L}_{\odot}\right.$, $\left.T_{\text {eff }}=34680 \mathrm{~K}\right)$. For an observer this motion pattern leads to periodic luminosity variations with $P=1.611$ days whereas the internal pulsation period is close to 0.8 days (see text for more details).

\section{Atmospheres of pulsating LBVs}

The non-linearities of the pulsations increase with the mass of the star and the effects described in this section will occur for masses above $30 \mathrm{M}_{\odot}$. As already seen in the light curves (Fig. 1) and the mass shells (Fig. 2) the at- 
mospheric motions are characterized by the shock waves leading to prominent perturbations of the outgoing stellar radiation. Since the waves deposit energy and momentum into the outermost stellar regions, the whole density and temperature stratification changes. Consequently, the location of the photosphere defined through

$$
L=4 \pi \sigma R^{2} T_{\mathrm{eff}}^{4}
$$

can be shifted by large values. Taking the mean value over a pulsational cycle, the effective temperature changes e.g. for model M60C from the initial hydrostatic value of $T_{\text {eff }}=18000 \mathrm{~K}$ to $20700 \mathrm{~K}$. Caused by the large amount of radiation pressure, the density inversion of the hydrostatic model cannot be maintained in dynamical models, which allows one to see deeper into the stellar atmosphere. The non-monotonic gradients in the dynamic atmospheres also permit sudden frequency-dependent changes of the optical depth meaning that not all the radiation leaving the star has to come from the same atmospheric layer. Both effects, the compression of the gas by the shock waves as well as the non-monotonic behavior of the photosphere, are responsible for the complicated structure of the light curves (Fig. 1). However, as demonstrated in Fig. 2 the underlying mass motions can exhibit a much simpler pattern.

Figure 3 illustrates these changes of the observable parameters comparing a hydrostatic with a dynamical atmosphere in the case of a pulsating LBV with $60 \mathrm{M}_{\odot}$. The thick solid line represents the initial model where the dominance of the radiative forces (Fig. 3, lower right panel) generate an unavoidable density and gas pressure inversion situated around $80 \mathrm{R}_{\odot}$ (Fig. 3, left panels). The grey shaded areas depict the upper and lower boundaries of physical quantities during a pulsational cycle. The resulting dynamic structure differs in many aspects from the hydrostatic stratification since outwards of $30 \mathrm{R}_{\odot}$ shock waves are propagating through the stellar layers. Correspondingly, the density, temperature and pressure stratification leads to a different appearance of such a pulsating star relative to a hydrostatic configuration. This dynamic atmosphere (outside of $30 \mathrm{R}_{\odot}$ for model $\mathrm{M} 60 \mathrm{C}$ ) exhibits radial structures, which cannot be encompassed by the initially hydrostatic structure which is based on static equilibrium conditions. In particular, the aforementioned density inversion can never be supported in such a dynamical environment. The effective photospheric radius shrinks from $R=97.4 \mathrm{R}_{\odot}$ to a mean value oscillating around $\bar{R}_{\text {dyn }}=74.2 \mathrm{R}_{\odot}$. Since the mean luminosity $L$ has to remain constant we get from Eq. (1) how the temperature of the dynamical atmospheres $T_{\text {dyn }}$ relates to the initial hydrostatic value of $T_{\text {eff }}$ by

$$
T_{\mathrm{dyn}}=T_{\mathrm{eff}} \sqrt{\frac{R}{\bar{R}_{\mathrm{dyn}}}} .
$$



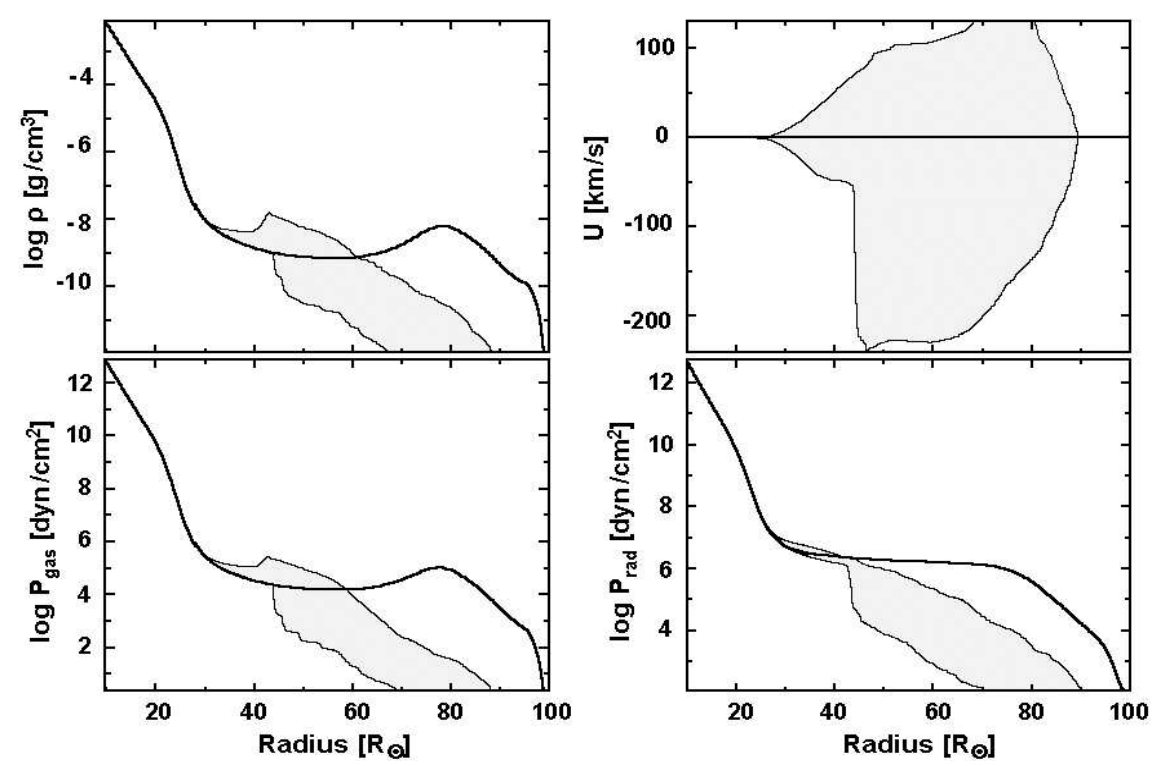

Figure 3: The difference between hydrostatic and hydrodynamic models for the model M60C $\left(M=60 \mathrm{M}_{\odot}, L=900000 \mathrm{~L}_{\odot}, T_{\text {eff }}=18000 \mathrm{~K}\right)$. The thick solid line plots the initial hydrostatic structure, the grey shaded areas show the variation of the density, velocity as well as gas and radiation pressure during a fully developed pulsational cycle.

Inserting the aforementioned values for the radii we obtain from the last equation $T_{\text {dyn }}=20620 \mathrm{~K}$ in agreement with the averaged value of the non-linear simulations of $20700 \mathrm{~K}$. Due to the fact that the atmosphere is extended, thin and rather transparent the main effect comes from the inward shift of the effective photosphere allowing such a simple description of an overall bluer appearance of the LBV. The shock waves running through the atmosphere with high Mach numbers (see $u_{\mathrm{s}}$ in Table 2) affect mainly the light curves as depicted in Fig. 1.

\section{Conclusions}

Summarizing a large number of non-linear pulsation computations (e.g. Dorfi et al. 2001) we emphasize that in general the observations of variable stars with large amplitude pulsations cannot be interpreted by simple hydrostatic atmospheric models. The stratification of the outer stellar layers is strongly modified by the shock waves, which heat and accelerate the atmospheric material. Although the small number of galactic LBVs makes it very unlikely that such 
stars will be observed within the COROT mission it should be pointed out that the deduced stellar parameters of stars with large amplitude pulsations have to analyzed through dynamical atmospheres and not by adopting hydrostatic model atmospheres.

The short life time of about $10^{4}$ years (based on statistics) of the LBV phenomenon makes observational studies more difficult. Nevertheless, it is necessary to obtain regular sampled photometric data with accuracies of the order of $0.01 \mathrm{mag}$ together with a time resolution of much less than one day. Since we have found very regular pulsating stars in the non-linear computations a careful monitoring would be essential to verify or disprove these predicted oscillations. If the observations of such stars are not done with an appropriate sampling the predicted pulsations can either be overlooked or interpreted as some kind of irregular flickering. To investigate the pulsational properties of LBVs well-sampled photometric data would be of great importance.

Acknowledgments. This project was supported by the Fonds zur Förderung der Wissenschaften (FWF) under project number S7305-AST.

\section{References}

Bohannan, B. 1997, in Luminous Blue Variables: Massive Stars in Transition, Eds.

A. Nota and H. J. G. L. M. Lamers, ASP Conf. Series Vol. 120, p.3

Dorfi, E. A. 1998, 27th Saas Fee Course, Springer, Berlin, p.263

Dorfi, E. A., Gautschy, A. 2000, ApJ 545, 982

Dorfi, E. A., Feuchtinger, M. U., Höfner, S. 2001, in Stellar Pulsation - Nonlinear

Studies, Eds. D. Sasselov and M. Takeuti, ASSL, Kluwer, Dordrecht, p.137

Eyer, L., Grenon, M. 1997, in HIPPARCOS - Venice ‘97, ESA SP-402, p.467

Gautschy, A., Saio, H. 1995, ARA\&A 33, 75

Kiriakidis, M., Fricke, K. J., Glatzel, W. 1993, MNRAS 264, 50

Leitherer, C. 1997, in Luminous Blue Variables: Massive Stars in Transition, Eds.

A. Nota and H. J. G. L. M. Lamers, ASP Conf. Series Vol. 120, p.58

Mihalas, D., Mihalas, B. W. 1984, Foundations of Radiation Hydrodynamics, Oxford University Press, New York

Nata, A., Lamers, H. J. G. L. M. (Eds.) 1997, Luminous Blue Variables: Massive

Stars in Transition, ASP Conf. Series Vol. 120

Shemmer, O., Leibowitz, E. M., Szkody, P. 2000, MNRAS 311, 698

Stothers, R. B., Chin, C.-W. 1993, ApJ 408, L85 